\title{
Effective shape and phase behavior of short charged rods
}

\author{
Eelco Eggen, ${ }^{1}$ Marjolein Dijkstra, ${ }^{2}$ and René van Roij ${ }^{1}$ \\ ${ }^{1}$ Institute for Theoretical Physics, Utrecht University, Leuvenlaan 4, 3584 CE Utrecht, The Netherlands \\ ${ }^{2}$ Soft Condensed Matter, Debye Institute for Nanomaterials Science, Utrecht University, \\ Princetonplein 5, 3584 CC Utrecht, The Netherlands \\ (Received 18 December 2008; published 2 April 2009)
}

\begin{abstract}
We explicitly calculate the orientation-dependent second virial coefficient of short charged rods in an electrolytic solvent, assuming the rod-rod interactions to be a pairwise sum of hard-core and segmental screenedCoulomb repulsions. From the parallel and isotropically averaged second virial coefficient, we calculate the effective length and diameter of the rods, for charges and screening lengths that vary over several orders of magnitude. Using these effective dimensions, we determine the phase diagram, where we distinguish a lowcharge and strong-screening regime with a liquid crystalline nematic and smectic phase, and a high-charge and weak-screening regime with a plastic crystal phase in the phase diagram.
\end{abstract}

DOI: 10.1103/PhysRevE.79.041401

PACS number(s): 82.70.-y, 64.70.pv, 64.70.M-, 82.35.Rs

\section{INTRODUCTION}

The study of suspensions of nonspherical colloidal particles started with the experimental works of Zocher [1] and Bawden et al. [2], and with Onsager's theoretical work [3]. It has since developed into a very versatile field of research. A lot of attention has been focused on needle-shaped rods, either naturally occurring ones such as viruses like tobacco mosaic virus or fd virus $[2,4,5]$, or laboratory synthesized ones such as Boehmite rods [6]. In recent years, however, a plethora of nonspherical particles have been synthesized that are not extremely elongated, for example, ellipsoidal colloids with aspect ratio $\sim 3$ [7], colloidal dumbbells [8], or nanoparticles with the shape of a rod, disk, snowman, cube, cap, or raspberry [9-15]. These particles are often charged when dissolved in a polar solvent such as water, and hence their pair interactions involve not only the anisotropic steric shortrange repulsions but also electrostatic long-range repulsions. The strength of the latter is determined by the charge on the particle and the range is determined by the Debye screening length of the solvent $[16,17]$. For small charges and strong screening (i.e., high salt concentrations), one expects the steric interactions to be dominant (if we assume that dispersion forces can be neglected). Hence, one can use computer simulations or theoretical studies of hard anisotropic bodies [18-23] to obtain an idea of the phase diagram of the system as a function of concentration. In the case of a high charge or weak screening (i.e., low salt concentration), however, the situation is less clear cut. There, the degree of anisotropy of the electrostatic interactions is not obvious from the outset: on the one hand one expects the soft screened-Coulomb interactions to wash out the hard-core anisotropy such that the interactions become effectively more spherically symmetric, while on the other hand there are the intriguing findings reported, for example, in Refs. [24,25]. The studies in these papers apply to systems of charged anisotropic particles in a screening medium. It was found that the electrostatic anisotropy persists to infinitely large distances as the asymptotic decay of each multipole contribution to the electrostatic potential due to a nonspherical charge distribution is equal $[24,25]$. This conclusion is in sharp contrast to the case of a charge distribution in vacuum, where the monopole potential decays more slowly than that of the dipole, as each order multipole contribution decays slower than the next one does. In our paper we investigate the interplay between hard-core and electrostatic interactions for nonspherical particles, for the relatively simple particle shape of spherocylinders.

It is well established by now that nonspherical colloidal particles can form a wealth of phases in thermodynamic equilibrium. Needlelike colloidal rods, for instance, form a phase sequence $I-N-S m-X$ upon increasing the concentration from very dilute up to close packing, where $I$ is the completely disordered isotropic fluid phase, $N$ the liquid crystalline nematic phase with orientational ordering, Sm the smectic- $A$ phase built from orientationally ordered liquidlike layers, and $X$ a fully ordered crystal phase $[1,3,4,18,26-30]$. This phase sequence for colloidal needles is well established for hard-core interactions [18,26,27]. Also, for softer electrostatic screened-Coulomb repulsions in the case of charged needles, at least in the regime where the length of the rods is much larger than the diameter and the screening length of the electrolytic solvent. This ensures that the effective diameter of the rods is much smaller than the length $[4,31]$. By contrast, particles with shapes that are sufficiently close to spherical are not expected to exhibit the liquid crystalline phases $N$ and Sm due to their small anisotropy. Instead, for such near-spherical particles one would expect a plastic crystalline phase $(P)$ to appear in the phase diagram, residing in between the isotropic fluid and the fully ordered crystal. The $P$ phase is characterized by positional ordering on a lattice, but without long-ranged orientational ordering of the particles. For instance, a phase sequence $I-P-X$ upon increasing the concentration has indeed been established in simulations of short hard spherocylinders and of hard dumbbells with a length-to-diameter ratio smaller than about $0.35[18,32,33]$. The question we address in this paper concerns the effect of colloidal charge and ionic screening on the effective shape of relatively short rods, and on their expected phase sequence upon increasing the concentration. On the basis of the well established increase of the effective diameter of charged needles compared to their hard-core diameter [31], it is to be expected that high colloidal charges and weak-screening conditions (i.e., low salt concentrations) lead to a decreased 
anisotropy of short charged rods. Hence, this will lead to a larger tendency of the system to exhibit a plastic crystal phase instead of liquid crystalline phases in the phase diagram, even if the hard-core shape would allow for liquid crystalline equilibrium phases.

Of course, suspensions of charged rods have been extensively studied theoretically before. Many of these studies are based on Onsager's second virial theory for hard rods [3], which is modified and extended to take into account the effects of charge and screening on the isotropic-to-nematic transition [31,34-38]. Some of these studies, for example, those of Refs. [3,31,34], focus on the needle limit in which the rod length is very large compared to the screening length. In this limit, only the diameter is affected by the electrostatic effects, but in such a way that the effective geometry of the rod remains needlelike. In Refs. [35,36] rod lengths of the order of (or larger than) the Debye length are considered, at the expense, however, of ignoring many of the prefactors such that the theory is essentially a scaling theory. Interestingly, this scaling theory predicts nematic-nematic coexistence in some parameter regime, which was later confirmed in Ref. [37]. This coexistence regime is characterized by a small rod charge density, such that the effective geometry of the rod is no longer needlelike. Another limit that was studied in detail is the limit of weak electrostatic interactions, which naturally leads to a perturbative description $[37,39,40]$. These schemes are very successful at describing the effective (non-needlelike) geometry that shows up in the angular dependence of the second virial coefficient. Another very interesting effect was identified in Ref. [38], where the correlation free energy of the many-body system of charged rods and counterions was calculated, resulting in an enhanced tendency to orientational ordering and also the possibility of nematic-nematic coexistence. With the notable exception of Ref. [40], however, most of these works on charged rods focus on the isotropic and nematic phases and hence, implicitly, on rods which are sufficiently elongated to give liquid crystalline phases at all.

In this paper we take a slightly different perspective. We explicitly calculate the orientation-dependent second virial coefficient of rather short charged rods numerically, for colloidal charges and screening lengths that vary over many decades. Such calculations, in which we use expansions in spherical harmonics, do not require only the asymptotic farfield expressions of the multipoles (such as considered in Refs. [24,25]), but in fact their full distance dependence. From the resulting second virial coefficient, we determine an effective hard-core length and diameter. Subsequently, we use these-in combination with the published hard-core phase diagram [18] — to determine the expected phase sequence upon increasing the concentration. This scheme is too crude to distinguish subtleties such as whether or not there is a nematic-nematic coexistence regime or to what extent the isotropic-nematic phase gap is affected. However, it is supposed to indicate reliably whether liquid crystalline ( $N$ and $\mathrm{Sm})$ or plastic crystal $(P)$ phases are to be expected in between the isotropic $(I)$ and crystalline $(X)$ phase. We focus on the case where the rod length is of the order of the screening length or smaller, in contrast to most of the previous theoretical work. This is the regime where the crossover from $N$ and
Sm to $P$ is expected to occur. In the limit where the rod length is small and the hard-core interactions are important, we give a simplified theoretical description that turns out to be in remarkable agreement with the numerical results. As our numerical approach relies on an expansion in spherical harmonics of the effective pair interaction between two rods, it leads to explicit but involved expressions. We present some of the mathematical technicalities of the derivation of these expressions in the Appendixes.

\section{MODEL}

We consider a system of identical charged colloidal rods suspended in an electrolyte solvent of dielectric constant $\epsilon$, Debye screening length $\kappa^{-1}$, and Bjerrum length $l_{\mathrm{B}}$ $=e^{2} /\left(4 \pi \epsilon k_{\mathrm{B}} T\right)$, at temperature $T$. Here $e$ is the elementary charge, and $k_{\mathrm{B}}$ is the Boltzmann constant. The rods are assumed to have the shape of a spherocylinder consisting of a cylinder of length $L$ and diameter $D$ capped by two hemispheres also of diameter $D$. The rods have a fixed charge, which we treat here as an (effective) line-charge density $e \lambda$ distributed homogeneously on the axis of the cylinder. We are interested in the effective pair potential $V\left(\mathbf{r} ; \hat{\omega}, \hat{\omega}^{\prime}\right)$ between two rods with orientations $\hat{\omega}$ and $\hat{\omega}^{\prime}$ at a center-tocenter vector $\mathbf{r}$, thermally averaged over the degrees of freedom of the electrolyte solvent (characterized by $\kappa^{-1}$ and $l_{\mathrm{B}}$ ). In the spirit of Derjaguin, Landau, Verwey, and Overbeek (DLVO), we assume that the effective pair potential consists of steric hard-core repulsions and electrostatic screenedCoulomb interactions between segments of the line charge of the two rods. We ignore short-ranged van der Waals attractions (i.e., we assume the particle and the solvent to be index matched or that the dispersion forces are canceled by steric or charge stabilization). Within these approximations the effective pair potential can be written as

$$
\beta V\left(\mathbf{r} ; \hat{\omega}, \hat{\omega}^{\prime}\right)= \begin{cases}\infty & \text { for overlapping rods, } \\ \beta V_{\mathrm{e}}\left(\mathbf{r} ; \hat{\omega}, \hat{\omega}^{\prime}\right) & \text { otherwise, }\end{cases}
$$

where $\beta^{-1}=k_{\mathrm{B}} T$, the overlap refers to the hard-core repulsions, and the electrostatic interaction potential is given by

$\beta V_{\mathrm{e}}\left(\mathbf{r} ; \hat{\omega}, \hat{\omega}^{\prime}\right)=l_{\mathrm{B}} \lambda^{2} \int_{-L / 2}^{+L / 2} d l \int_{-L / 2}^{+L / 2} d l^{\prime} \frac{\exp \left[-\kappa\left|\mathbf{r}+l^{\prime} \hat{\omega}^{\prime}-l \hat{\omega}\right|\right]}{\left|\mathbf{r}+l^{\prime} \hat{\omega}^{\prime}-l \hat{\omega}\right|}$.

The integration variables $l$ and $l^{\prime}$ play the role of coordinates running along the cylinder axis of each of the two rods, from one end of the cylinder to the other end. In the long-rod limit, $L / D \gg 1$ and $\kappa L \gg 1$, one can replace the integration domains in Eq. (2) by the full real axis, together with the constraint that the cylinder axes are in "cross configuration" (i.e., the axes intersect when projected onto the plane parallel to both axes). Otherwise, the potential vanishes. One then easily shows that $V_{\mathrm{e}}$ only depends on the shortest distance and the relative angle between the two rods $[3,31,37]$. Here we focus on shorter rods, for which this simplification does not apply. In the Appendixes we derive systematic series 
expansions in spherical harmonics to describe the angular and position dependence of $V_{\mathrm{e}}$ explicitly, focusing on rods that are rather short compared to the Debye screening length (which sets the range of the electrostatic repulsions). More specifically, the expansion of the angular dependence is truncated and we consider each term as an expansion in $\kappa L$ up to fourth order (see Appendix E). We compare the result with the large- $\kappa L$ limit.

The present model can be characterized by a few dimensionless combinations. In the limit of uncharged rods $(\lambda$ $=0$ ), the aspect ratio $L / D$ of the hard-core dimensions is of primary importance. However, for the charged rods of present interest, the ratio $\kappa L$ (of the hard-core length to the Debye screening length of the solvent) gives more information on the interaction anisotropy. The ratio $\kappa D$ is relevant as a measure of ionic strength. Dimensional inspection of the expression in Eq. (2) shows that the strength of the electrostatic interactions is determined by the dimensionless (square of the) line charge density

$$
q \equiv \frac{l_{\mathrm{B}} \lambda^{2}}{\kappa} .
$$

These dimensionless combinations can span quite a range of numerical values in experimental systems. For instance, for fd virus suspended in water one finds [5] $L / D>100, \kappa D$ $\simeq 0.1-1$ and $q=70-700$, and recently synthesized silica dumbbells in oily solvents [41] are best characterized by $L / D \simeq 1, \kappa D \simeq 1$, and $q \simeq 10^{2}$. Short (double stranded) DNA chains have $\kappa D \simeq 0.1-1$ and $q \simeq 0.1-10$, while their length can be varied by the number of base pairs included in the sequence. These chains can be characterized as rigid rods up to the persistence length corresponding to $L / D \simeq 50$. Moreover, present-day synthesis techniques allow for the tuning of surface charge, in principle at least, from essentially vanishing to extremely high. This is achieved, for example, by using different coatings with varying degrees of ion dissociation of the surface groups. It is therefore of interest to investigate the thermodynamics of the present model over a wide range of parameters.

\section{THERMODYNAMICS AND EFFECTIVE DIMENSIONS}

With the pair potential specified by Eqs. (1) and (2), and with an explicit scheme to evaluate it as explained in the Appendixes, we can study the macroscopic properties of suspensions of these charged rods. In principle, we do this as a function of concentration, for various $q, \kappa D$, and $L / D$. Here we circumvent the complexity of the full statisticalmechanical calculation of free energies and phase diagrams of the system at hand. We do this by mapping the second virial coefficient of the charged spherocylinders of interest onto that of hard spherocylinders with an effective cylinder length $L_{\text {eff }}$ and an effective diameter $D_{\text {eff }}$ that we will calculate below. We then presume that the phase diagram of the system of charged rods follows from that of the effective hard-rod system, which we take from published computer simulation data [18]. It is well known from these and follow-up simulations of hard rods, as well as density functional theory $[26,27]$, that this system exhibits a sequence of

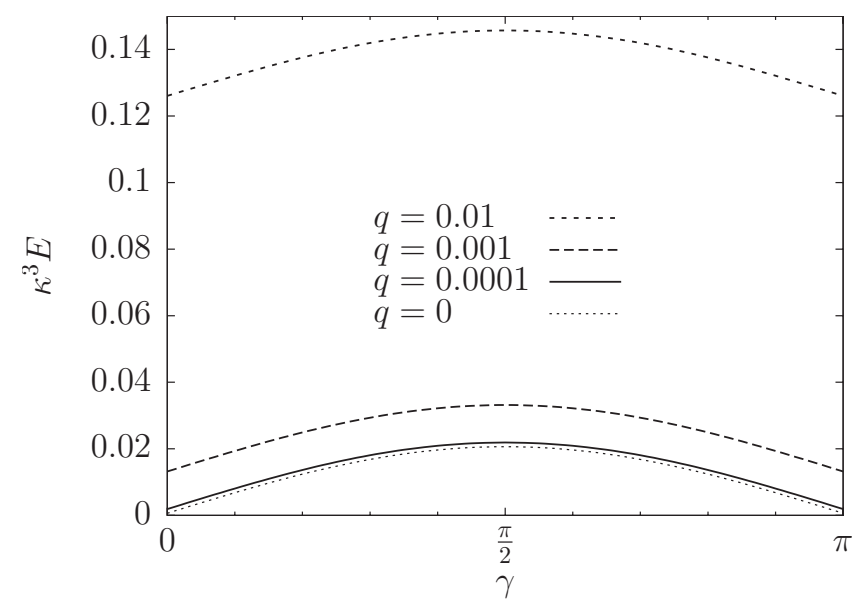

FIG. 1. The effective excluded volume $\kappa^{3} E$, as a function of the angle $\gamma$ between the two rod orientations, for different values of the charge parameter $q$. We used the parameter values $\kappa L=1$ and $\kappa D$ $=0.01$ (such that $L / D=100)$.

phase transitions upon increasing the concentration that strongly depends on the aspect ratio $L / D$ : sufficiently elongated hard rods with $L / D>3.7$ have an isotropic-nematicsmectic-crystal $(I-N-\mathrm{Sm}-X)$ phase sequence, sufficiently short hard rods $0<L / D<0.35$ show an $I-P-X$ sequence with $P$ a plastic crystal, and in between there are two more regimes in which the $N$ and $P$ phase, respectively, no longer appear in the phase sequence. Below we determine how the analogous crossovers between these regimes of the effective system, as determined by $L_{\text {eff }} / D_{\text {eff }}$, depend on the parameters $q, \kappa D$, and $L / D$.

A key ingredient of our calculation is the effective excluded volume $E\left(\hat{\omega}, \hat{\omega}^{\prime}\right)$ of two charged rods with orientations $\hat{\omega}$ and $\hat{\omega}^{\prime}$, defined as

$$
E\left(\hat{\omega}, \hat{\omega}^{\prime}\right)=\int d \mathbf{r}\left(1-\exp \left[-\beta V\left(\mathbf{r} ; \hat{\omega}, \hat{\omega}^{\prime}\right)\right]\right),
$$

where the pair potential between the rods is given in Eqs. (1) and (2). Note that $E\left(\hat{\omega}, \hat{\omega}^{\prime}\right)$ is in fact twice the corresponding second virial coefficient, and that the nomenclature "effective excluded volume" stems from the fact that it reduces to the actual excluded volume of the pair in the case of purely hard-core interactions. On the basis of symmetry arguments one easily checks that the angular dependence of $E\left(\hat{\omega}, \hat{\omega}^{\prime}\right)$ is in fact only through the angle $\gamma=\arccos \left(\hat{\omega} \cdot \hat{\omega}^{\prime}\right)$ between the cylinder axes of the two rods. In Fig. 1 we show this $\gamma$ dependence of $E$ for rods characterized by $\kappa L=1$ and $\kappa D$ $=0.01$ (so $L / D=100$ and weak screening), for several charge parameters $q$ ranging from $q=0$ (uncharged) to $q=0.01$ (fairly charged). The results of Fig. 1 stem from a combination of numerical and analytic procedures explained in detail in the Appendixes. These involve a fivefold integration: over the contour of the rods $l$ and $l^{\prime}$ in Eq. (2), and the center-tocenter separation vector $\mathbf{r}$ in Eq. (4).

The key observations of Fig. 1, which is typical for many system parameters, are that for increasing $q$ the effective excluded volume becomes (i) less anisotropic, and (ii) larger in magnitude. Moreover, for all $q$ the effective excluded vol- 
ume is larger for perpendicular orientations than for parallel ones. Qualitatively, and in fact quantitatively for many parameters, this behavior is identical to that of hard spherocylinders of effective length $L_{\text {eff }}$ and diameter $D_{\text {eff }}$, for which the excluded volume is given by [3]

$$
\mathcal{V}_{\text {eff }}(\hat{\omega}, \hat{\omega})=\frac{4 \pi}{3} D_{\text {eff }}^{3}+2 \pi L_{\text {eff }} D_{\text {eff }}^{2}+2 L_{\text {eff }}^{2} D_{\text {eff }} \sin \gamma
$$

In principle one can fit the functional form of Eq. (5) to the numerical results such as those of Fig. 1 to determine the effective hard-core dimensions $L_{\text {eff }}$ and $D_{\text {eff }}$ for given charged-rod parameters. However, instead of fitting the full angular dependence numerically, it is more convenient to match the isotropically averaged effective excluded volume and the parallel one, given by

$$
E_{\mathrm{iso}}=\frac{1}{(4 \pi)^{2}} \int d \hat{\omega} \int d \hat{\omega}^{\prime} E\left(\hat{\omega}, \hat{\omega}^{\prime}\right)=\frac{1}{2} \int_{0}^{\pi} d \gamma \sin \gamma E(\gamma)
$$

$$
E_{\|}=E(\hat{\omega}, \hat{\omega})=E(\gamma=0),
$$

to the values for spherocylinders with effective hard-core dimensions $L_{\text {eff }}$ and $D_{\text {eff }}$

$$
\begin{gathered}
\mathcal{V}_{\mathrm{iso}}=\frac{4 \pi}{3} D_{\mathrm{eff}}^{3}+2 \pi L_{\mathrm{eff}} D_{\mathrm{eff}}^{2}+\frac{\pi}{2} L_{\mathrm{eff}}^{2} D_{\mathrm{eff}}, \\
\mathcal{V}_{\|}=\frac{4 \pi}{3} D_{\mathrm{eff}}^{3}+2 \pi L_{\mathrm{eff}} D_{\mathrm{eff}}^{2},
\end{gathered}
$$

respectively. This procedure yields the effective hard-core dimensions

$$
\begin{gathered}
D_{\text {eff }}=\left[\frac{3 E_{\|}}{4 \pi}(1+3 \Delta-\sqrt{3 \Delta(2+3 \Delta)})\right]^{1 / 3}, \\
\frac{L_{\text {eff }}}{D_{\text {eff }}}=2 \Delta+\frac{2}{3} \sqrt{3 \Delta(2+3 \Delta)},
\end{gathered}
$$

where we used, for notational convenience, the dimensionless anisotropy parameter $\Delta$ defined as

$$
\Delta \equiv \frac{E_{\mathrm{iso}}-E_{\|}}{E_{\|}}
$$

It turns out that inserting $L_{\text {eff }}$ and $D_{\text {eff }}$ as obtained from Eqs. (10)-(12) into Eq. (5) gives an angular dependence that is in very good agreement with the numerically obtained effective excluded volume of charged rods.

It is also interesting to compare our numerical results with analytic expressions that are valid in the limit where $L / D$ $\gg 1$ and $\kappa L \gg 1$, as obtained by Stroobants et al. [31]. In this needle limit the effective excluded volume is given by

$$
\begin{aligned}
E_{\infty}(\gamma)= & 2 L^{2} \kappa^{-1} \sin \gamma\left[\gamma_{\mathrm{E}}+\ln 2 \pi q-\ln \sin \gamma\right. \\
& \left.+\Gamma\left(0, \frac{2 \pi q \exp [-\kappa D]}{\sin \gamma}\right)\right],
\end{aligned}
$$

where $\gamma_{\mathrm{E}} \approx 0.577$ is the Euler-Mascheroni constant and where the incomplete gamma function (or exponential integral) is defined by

$$
\Gamma(\alpha, x)=\int_{x}^{\infty} d y y^{\alpha-1} \exp [-y] .
$$

From this expression-using the Onsager limit $\mathcal{V}_{\text {iso, } \infty}$ $=(\pi / 2) L^{2} D_{\text {eff, } \infty}$ for the isotropically averaged excluded volume- the effective diameter can be calculated

$$
\begin{aligned}
\kappa D_{\mathrm{eff}, \infty}= & \gamma_{\mathrm{E}}+\ln 2 \pi q+\ln 2-\frac{1}{2} \\
& +\frac{2}{\pi} \int_{0}^{\pi} d \gamma \sin ^{2} \gamma \Gamma\left(0, \frac{2 \pi q \exp [-\kappa D]}{\sin \gamma}\right) .
\end{aligned}
$$

The effective length is taken equal to the rod length $L_{\mathrm{eff}, \infty}$ $=L$.

\section{NUMERICAL RESULTS}

Calculations such as those of Fig. 1 are reasonably accurate for values of $\kappa L$ roughly up to 2 . For higher values the applied approximations become poor, such that for $\kappa L \gtrsim 3$ the calculations become even qualitatively unreliable for many of our parameters. For this reason we restrict most of our attention to the regime where $\kappa L \leqslant 2$.

In order to assess the accuracy of our calculations, we compare some of the results of our calculations with those obtained from more extensive numerical integration

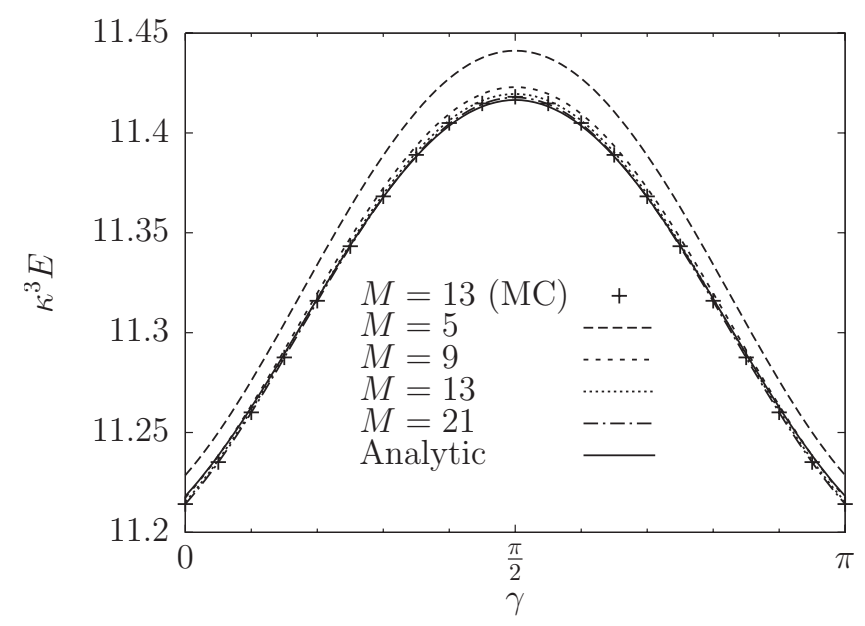

FIG. 2. The effective excluded volume $\kappa^{3} E$, as a function of the angle $\gamma$ between the rod orientations, calculated using different numerical schemes (see text), involving $M$ discrete charges, Monte Carlo (MC) integration, and the present analytic approach. We used the parameter values $\kappa L=1, \kappa D=0.25$ (such that $L / D=4$ ), and $q$ $=1$. 



FIG. 3. The effective diameter $\kappa D_{\text {eff }}$ as a function of the rod diameter $\kappa D$ for (a) $\kappa L=1$ and different values for the charge parameter $q$; (b) $q=10$ and different values for the rod length $\kappa L$. The rod dimensions are scaled by the screening length $\kappa^{-1}$. The thin solid line is a guide to the eye, representing the hard-core limit $D_{\text {eff }}=D$. The small solid circles give the values for $\kappa D=0$ from the numerical calculations. The larger open circles are obtained by the approximation given in Eq. (17).

schemes. One is given by the same spatial integration scheme as before, but with the (effective) line-charge density replaced by a discrete charge distribution. The rod charge is represented by an odd number of charge units $(M=2 N+1)$ distributed evenly on the cylinder axis, where one unit is always located on the center of the axis, and two units are always located on the two end points of the axis. The latter are of magnitude $e \lambda L /(4 N)$, while all others are of magnitude $e \lambda L /(2 N)$. This ensures that the total charge is $e \lambda L$ and the continuum limit $N \rightarrow \infty$ yields the correct homogeneous line charge. The other scheme uses the same discrete charge density as described above, but uses a Monte Carlo (MC) scheme to perform the integration. This scheme is denoted by the pluses in Fig. 2. The agreement between the results obtained from the different schemes, as shown in Fig. 2, is excellent for $M \geqslant 13$, particularly when considering that the shape of the effective excluded volume differs significantly from the hard-core case for these parameters. Therefore, we conclude that our calculation correctly predicts the angular dependence of the effective excluded volume of short charged rods.

In the previous section, we have shown that the angular dependence of the effective excluded volume can be used to calculate the effective rod dimensions $L_{\text {eff }}$ and $D_{\text {eff }}$-from the values of $E_{\|}$and $E_{\perp}$ - by applying Eqs. (10)-(12). Figure 3(a) shows the numerically calculated effective diameter as a function of the real diameter for $\kappa L=1$ and a range of charge parameters $q$. Figure 3(b) shows the same function, but then for $q=10$ and a range of rod lengths $\kappa L$. Note that all (effective) rod dimensions are expressed in units of the screening length. In Fig. 3(b) the needle limit $\kappa L \gg 1$, given by Eq. (15), is plotted for comparison. Both graphs clearly reveal two regimes,

$$
D_{\mathrm{eff}} \simeq \begin{cases}D_{\mathrm{e}} & \text { for } D \ll D_{\mathrm{e}}, \\ D & \text { for } D \gg D_{\mathrm{e}} .\end{cases}
$$

These can be identified as an electrostatic regime at small $\kappa D$ (weak screening) and a hard-core regime at high enough $\kappa D$ (strong screening). In the hard-core regime, the effective diameter equals the hard-core diameter, while in the (weakly screened) electrostatic regime the effective diameter saturates to a plateau value $D_{\mathrm{e}}$. This electrostatic effective diameter depends on $q$ and $\kappa L$, and increases with increasing $q$ and $\kappa L$. Also, it is (much) larger than the hard-core diameter due to the (strong) rod-rod repulsions. Values of the electrostatic effective diameter are included in Fig. 3, where the small solid circles represent values obtained from numerical calculations for $\kappa D=0$. The larger open circles represent the following simple approximation for $D_{\mathrm{e}}$.

In the short-rod limit, we can treat the double layer around the rod as spherically symmetric, with an effective point charge $e \lambda L$ in the center, such that also the pair potential is spherically symmetric. This gives $\Delta=0$, and hence from Eq. (10), for large enough $q$ (or small enough $\kappa D$ ), we obtain the electrostatic effective diameter from the simple expression

$$
\kappa D_{\mathrm{e}} \simeq\left[3 \int_{0}^{\infty} d x x^{2}\left(1-\exp \left[-q \kappa^{2} L^{2} \frac{\exp [-x]}{x}\right]\right)\right]^{1 / 3} .
$$

This approximation is given in Fig. 3 by the larger open circles. Both graphs show good agreement for $\kappa L \leqslant 1$ and all values for $q$. Figure 3(b) also shows that the regime $\kappa L \leqslant 2$-which is reliably accessible with our truncated numerical scheme-evolves smoothly to the needle-limit $\kappa L$ $\gg 1$ of Stroobants et al. [31]. The curve for $\kappa L=3$ shows some signatures of the numerical instabilities we encounter for larger $\kappa L$.

In a similar fashion we can also study the effective length $L_{\text {eff }}$ of the rods. Figure 4(a) shows results of numerical calculations of the effective rod length for $\kappa L=1$ and a range of charge parameters $q$. Figure 4(b) is the result for $q=10$ and a range of rod lengths $\kappa L$. The rod dimensions are expressed in units of the Debye length, whereas $L_{\text {eff }}$ is expressed in units of the hard-core length. We distinguish again two asymptotic regimes, the strong-screening (hard-core) regime $\kappa D \gg 1$ 



FIG. 4. The effective length $L_{\text {eff }} / L$ as a function of the rod diameter $\kappa D$ for (a) $\kappa L=1$ and different values for the charge parameter $q$; (b) $q=10$ and different values for the rod length $\kappa L$. The rod diameter is scaled by the screening length $\kappa^{-1}$ and the effective length is scaled by the rod length $L$.

where $L_{\mathrm{eff}}=L$, and the weak-screening (electrostatic) regime $\kappa D \ll 1$ where $L_{\text {eff }}$ reaches a plateau value that depends on $q$ and $\kappa L$. Note also that $L_{\text {eff }}<L$ which is perhaps unexpected at first sight. Naively, one could expect the effective length to increase with increasing effective excluded volume. However, as Sato and Teramoto [34] pointed out, the effective length decreases with increasing rod charge density because of end effects. Thus, the increase of the effective excluded volume - due to the increase of the rod charge density-is purely caused by the increase of the effective diameter. Moreover, this increase balances the decreasing in effective length such that the total effective particle length $L_{\text {eff }}+D_{\text {eff }}$ does increase with increasing rod charge density. Inspection of Fig. 4(a) also reveals numerical (convergence) problems for $q \geqslant 100$ at $\kappa D \geqslant 1$, where $\kappa L_{\text {eff }}$ sharply drops and rises before reaching the hard-core limit $L_{\text {eff }}=L$. This is in fact only a minor problem in practice, as it only occurs in the regime where $L_{\text {eff }} / D_{\text {eff }} \lesssim 0.1$. There, the anisotropic contribution to the effective excluded volume is much smaller than the isotropic part. Upon approach of the needle-limit $\kappa L$ $\gg 1$, see Fig. 4(b), we find that $L_{\text {eff }}$ approaches $L$ for all values of $\kappa D$, as expected.

\section{PHASE BEHAVIOR}

We have determined the effective length and diameter of charged rods, by mapping their orientation-dependent second virial coefficient onto that of effective hard rods. Subsequently, we also study the effective length-to-diameter ratio


function of the rod charge for $\kappa L=1$ and a range of rod diameters $\kappa D$. All curves with $\kappa D>0$ essentially decrease from their maximum value-the hard-core aspect ratio $L / D$-towards the curve given by $\kappa D=0$. This indicates that the effective dimensions of charged rods become independent of the hard-core diameter for large charge parameters, where we enter the electrostatic regime. Also, since the effective aspect ratio for $\kappa D=0$ is a decreasing function for large $q$, we see that the charged rods essentially behave as charged spheres upon increasing the charge above a certain value.
Moreover, Fig. 5 reveals a local maximum for very small $\kappa D$, in the regime where $q \simeq 1$. This effect can be understood by considering the electrostatic regime for small charge parameters $q$. Equation (11) shows that the effective aspect ratio is governed by the dimensionless anisotropy parameter $\Delta$, which is defined in Eq. (12). In the electrostatic regime, this anisotropy can be shown - up to first order-to be proportional to $q$. The reason for this is that the linear approximation of the effective excluded volume is orientation independent [37]. Therefore, the difference between the isotropically averaged and parallel values is of second order in $q$, whereas the parallel value itself is of first order. The effective aspect ratio is of order $\sqrt{\Delta}$, and thus increases as $\sqrt{q}$. Conversely, for $q \gtrsim 1$ the effective length is more or less constant, and the effective aspect ratio decreases again due to the increase of the effective diameter.

The horizontal dotted lines in Fig. 5 indicate the crossover values $(0.35,3.5$, and 3.7$)$ for regimes with different phase



FIG. 5. The effective aspect ratio $L_{\text {eff }} / D_{\text {eff }}$ as a function of the charge parameter $q$ for $\kappa L=1$ and different values for the rod diameter $\kappa D$. Different-possibly coexisting-phases are associated with a certain range of (effective) aspect ratios. See the text for an explanation of the abbreviations and the boundary values. 



FIG. 6. Boundary lines for given values for the effective aspect ratio $L_{\mathrm{eff}} / D_{\mathrm{eff}}$. See the text for an explanation of the abbreviations of the different regime labels. The points are results of the numerical calculations, and the lines are given by a simplified theory. We fix (a) $\kappa L$ $=1$, and (b) $L / D=20$, respectively.

sequences. The values for these aspect ratios are taken from simulation results of hard-spherocylinder systems by Bolhuis and Frenkel [18]. These simulations consist of explicit freeenergy calculations of coexisting phases, where the most dilute phase is always given by an isotropic fluid (I), and the most dense phase by a fully ordered crystal $(X)$. Depending on the aspect ratio, different phases were found in between these two phases. For aspect ratios exceeding $\sim 3.7$ the phase sequence $I-N-\mathrm{Sm}-X$ was found upon increasing the density. Here, the $N$ and Sm denote the nematic and smectic- $A$ liquid crystalline phases, respectively. Somewhat shorter rods, with an aspect ratio in the narrow regime between $\sim 3.5$ and $\sim 3.7$, can still form a smectic- $A$ but no longer a nematic phase, and hence have a phase sequence $I$-Sm- $X$. Even shorter hard rods, with an aspect ratio in between $\sim 0.35$ and $\sim 3.5$ cannot form a thermodynamically stable smectic- $A$ phase, and thus crystallize directly into a fully ordered crystal from the isotropic fluid, yielding a phase sequence $I-X$. Very short hard rods, with an aspect ratio smaller than $\sim 0.35$, exhibit a plastic $(P)$ crystal phase, such that the phase sequence is $I-P-X$. The plastic crystal phase is characterized by orientational disorder, but has translational order as in a crystal phase $[18,32]$. This regime arises naturally in the case that $\kappa L$ is small. Then, such a crystal forms because of the essentially isotropic long-range repulsive interactions, but the competition with entropic effects prevents the rods from aligning.

We use the mapping of the charged-rod system onto the effective hard-rod system to give an indication of the phase sequence of systems of charged rods as a function of the parameters $\kappa L, \kappa D($ or $L / D)$, and $q$. For instance, from the curve for $\kappa D=1$ in Fig. 5, we see that the effective aspect ratio never exceeds unity for any $q$. This excludes the possibility of a nematic or smectic- $A$ liquid crystal phase. The curve starts off at its maximum (in the limit where $q \rightarrow 0$ ), where the effective aspect ratio equals the hard-core aspect ratio $L / D=\kappa L=1$. It crosses the value $L_{\text {eff }} / D_{\text {eff }}=0.35$ at $q$ $\approx 2.35$, such that a sufficiently large rod charge density al- lows for a plastic crystal phase. Similarly, for $\kappa D=0.1$ (which corresponds to $L / D=10$ ), we find all four phase sequences upon increasing $q$.

By determining the intersections of the effective aspect ratio with the crossover values of the hard-rod system, we construct "phase diagrams" indicating the different regimes. In Fig. 6 we present two examples of such diagrams in the plane spanned by $q$ and $\kappa D$. In Fig. 6(a), we fix $\kappa L=1$, such that the horizontal axis could read $D / L$ as well. In Fig. 6(b) we fix $L / D=20$, such that the change in $\kappa D$ physically corresponds to a change in salt concentration (while keeping the particle dimensions fixed). The symbols denote the crossover values for the effective aspect ratio as determined from our numerical data (such as presented in Fig. 5). The lines are based on an approximate theoretical model to be discussed in Sec. VI.

Both diagrams in Fig. 6 show that rods with sufficiently high surface charge density always show the $I-P-X$ sequence. This is due to the essentially spherical nature of the effective shape of highly charged rods. The limit of uncharged rods is determined by the hard-core sequence that corresponds to $L / D$. The $I-N-\mathrm{Sm}-X$ regime at fixed $\kappa L$ in Fig. 6(a) is completely bounded. First, by a hard-core regime when $\kappa D$ $\gtrsim 0.27$, where the liquid crystal phases cannot exist even for $q=0$ because $L / D \lesssim 3.7$. Second, by an electrostatic regime in the weak-screening limit of small $\kappa D$, where the rods effectively behave as spheres since $D_{\text {eff }} \gg L_{\text {eff }}$. Conversely, the trends displayed for fixed $L / D$ in Fig. 6(b) are monotonic, with an $I-N-\mathrm{Sm}-X$ regime that extends to higher $q$ with increasing $\kappa D$.

\section{A SIMPLER MODEL}

For small values of the effective surface-charge density, we found that the electrostatic contribution to the effective excluded volume is essentially isotropic in nature. This means that the anisotropic effects are primarily due to the hard-core anisotropy (as apparent from Fig. 1), such that 


$$
L_{\text {eff }}^{2} D_{\text {eff }} \simeq L^{2} D .
$$

On this basis, we propose here a simple model, which turns out to describe our numerical findings with remarkable accuracy. This model introduces a "spherical approximation" of the electrostatic contribution to the effective excluded volume, which involves the orientation-dependent diameter $\bar{D}(\gamma)$. The volume of a sphere of this diameter is equal to the hard-core excluded volume of a pair of rods

$$
\frac{4 \pi}{3} \bar{D}(\gamma)^{3}=\frac{4 \pi}{3} D^{3}+2 \pi L D^{2}+2 L^{2} D \sin \gamma
$$

We approximate the effective excluded volume by the value for a charged sphere of diameter $\bar{D}(\gamma)$ and an effective surface charge that equals the total amount of effective charge on the rods

$$
\begin{aligned}
\bar{E}(\gamma)= & \frac{4 \pi}{3} \bar{D}(\gamma)^{3}+4 \pi \int_{\bar{D}(\gamma)}^{\infty} d r \\
& \times r^{2}\left(1-\exp \left[-q \kappa^{2} L^{2} \frac{\exp [-\kappa r]}{\kappa r}\right]\right) .
\end{aligned}
$$

Note that the only orientation dependence of the electrostatic contribution to this effective excluded volume (i.e., the second term) comes from the integral boundary $\bar{D}(\gamma)$. To calculate the effective dimensions, we only need the parallel and isotropically averaged values of the effective excluded volume. In the parallel case $(\gamma=0)$ this value is readily calculated

$$
\bar{E}_{\|}=\frac{4 \pi}{3} \bar{D}_{\|}^{3}+4 \pi \int_{\bar{D}_{\|}}^{\infty} d r r^{2}\left(1-\exp \left[-q \kappa^{2} L^{2} \frac{\exp [-\kappa r]}{\kappa r}\right]\right),
$$

where

$$
\frac{4 \pi}{3} \bar{D}_{\|}^{3}=\frac{4 \pi}{3} D^{3}+2 \pi L D^{2} .
$$

The isotropically averaged value can be calculated numerically by using expression (20). However, we approximate it by the value for a charged sphere of diameter $\bar{D}_{\text {iso }}$ (using the same total effective charge), which is taken from the isotropic average of the hard-core excluded volume

$$
\frac{4 \pi}{3} \bar{D}_{\text {iso }}^{3}=\frac{4 \pi}{3} D^{3}+2 \pi L D^{2}+\frac{\pi}{2} L^{2} D .
$$

This approximation yields the simple expression

$$
\bar{E}_{\text {iso }}=\frac{4 \pi}{3} \bar{D}_{\text {iso }}^{3}+4 \pi \int_{\bar{D}_{\text {iso }}}^{\infty} d r r^{2}\left(1-\exp \left[-q \kappa^{2} L^{2} \frac{\exp [-\kappa r]}{\kappa r}\right]\right) .
$$

With our explicit expressions (21) and (24), we evaluate the effective dimensions from Eqs. (10) and (11) as before. The resulting crossover values of the hard-rod system are shown by the curves in Fig. 6, and are in very good agreement with the numerical calculations (denoted by the symbols). The key to this remarkable accuracy lies in the fact that the anisotropic electrostatic contributions are relatively unimportant, because the rod length is small with respect to the screening length (i.e., $\kappa L \leqslant 2$ ). Thus, our simple model accounts for the hard-core anisotropy correctly, as well as for the isotropic electrostatic contribution.

In a sense, this theoretical description can be viewed as a kind of perturbation theory, where we expand the pair potential as a function of $\kappa L$. The hard-core repulsion represents the zeroth order. The lowest-order contribution to $V_{\mathrm{e}}$ is quadratic in $\kappa L$ and independent of rod orientations. Also, it happens to correspond to the interaction potential of two point charges $e \lambda L$. If we plug this approximation of $V\left(\mathbf{r} ; \hat{\omega}, \hat{\omega}^{\prime}\right)$ into the expression of the effective excluded volume [given by Eq. (4)], we obtain an expression where the integral boundary $\bar{D}$ is still a function of both the angle between the rod orientations and the direction of the center-tocenter separation vector $\mathbf{r}$. In fact, it is given by the distance where the rods touch, given a certain orientational configuration. By setting this overlap diameter to a value that is independent of the orientation of $\mathbf{r}$, but still respects the total hard-core excluded volume, we effectively neglect its dependence on $\kappa L$. This choice is justified by the fact that (for small $\kappa L)$ the size of the double layer around the particles is larger than the variations in the overlap diameter $\bar{D}$. That is why our simple theoretical description can be interpreted as a perturbation theory of a hard-rod reference system with an (almost) isotropic electrostatic contribution. Unfortunately, it completely fails to describe the anisotropic effects in the electrostatic regime. In this regime the anisotropic details of the electrostatic contributions do become important compared to the hard-core contributions.

\section{DISCUSSION AND CONCLUSION}

The numerical results presented in this paper give access to a part of the parameter space where there is a large difference between the effective length and the real length. In this regime, one cannot hope that the theory of Stroobants et al. [31] gives any accurate results, as this is based on the needle limit where $L_{\mathrm{eff}} \simeq L$. The perturbation theory of Chen and Koch [37] breaks down for most of our parameter values. This is because it is based on small charges, and thus fails to describe the effect of large rod surface-charge densities. Also, this theory is not accurate for large differences between the effective and hard-core diameter.

In Fig. 7(a), we show results of numerical calculations of the effective rod length as a function of the hard-core length, for $\kappa D=0.1$. Note that again the effective length is always smaller than (or equal to) the hard-core length. Also, in accordance with the results from Fig. 4, there is a hard-core regime for small values of the charge parameter $q$, as well as for small values of the rod length $\kappa L$, for which the total amount of effective rod charge is small. On the other hand, there is an electrostatic regime. In Fig. 4, this was shown to be the case for decreasing values of $\kappa D$, where the plateau value (i.e., the electrostatic length) depends on $q$ and $\kappa L$. However, from Fig. 7(a), it can be seen that this electrostatic length depends mostly on the rod length $\kappa L$, and not really 

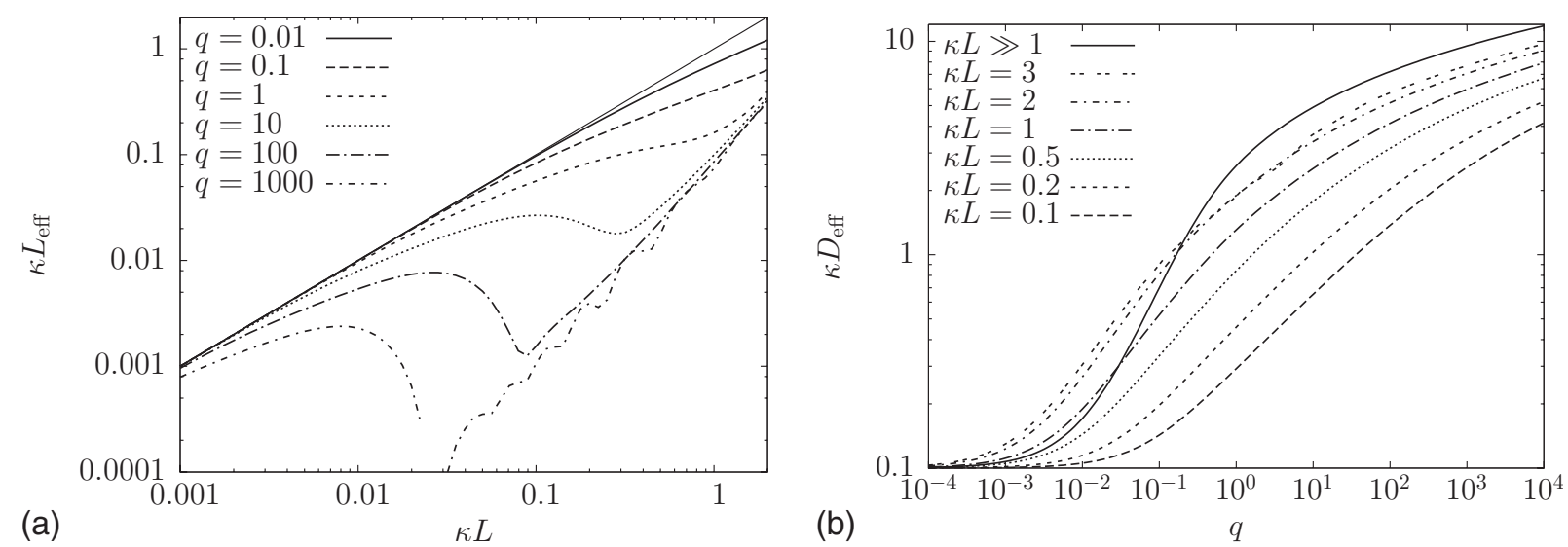

FIG. 7. (a) The effective length $\kappa L_{\text {eff }}$ as a function of the rod length $\kappa L$ for $\kappa D=0.1$ and different values for the charge parameter $q$. The thin solid line represents the needle or hard-core limit, where $L_{\text {eff }} \simeq L$. (b) The effective diameter $\kappa D_{\text {eff }}$ as a function of the rod charge parameter $q$ for $\kappa D=0.1$ and different values for the rod length $\kappa L$. The (effective) rod dimensions are scaled by the screening length $\kappa^{-1}$.

on the charge parameter $q$, as long as either $q$ or $\kappa L$ is large enough. Furthermore, the effective length is "wedged" in between the electrostatic length and the hard-core length, where the electrostatic length approaches the hard-core length in the needle limit $(\kappa L \gg 1)$.

Unfortunately, there is no analytic theory yet that describes our numerical results for this electrostatic length as a function of $\kappa L$. Therefore, it would be worthwhile to gain new insight in the effect of electrostatics on the effective rod length for intermediate $\kappa L$-neglecting hard-core interactions - in the case of large rod charges. In this regime, however, the angular dependence of the effective excluded volume is different from that of a hard spherocylinder. Visual inspection of Fig. 2 reveals this difference for small angles. Probably, the effective shape resembles more a hard ellipsoid of revolution, for which it is know that there is no stable smectic phase [42]. Thus, one can claim that the existence of a stable smectic phase is questionable in this regime. On the other hand, in the parameter regime that we focused on in this paper this subtlety is not directly relevant. The part of the diagram of phase sequences that shows a smectic phase is well described by the simplified model in which the anisotropy of the effective excluded volume purely stems from the hard-core interactions. In this case, the increase of $q$ leads to such a strong increase in the isotropic contribution to the effective excluded volume that the smectic phase disappears from the sequence. This happens before the onset of any significant change in the effective shape.

Additionally, Fig. 7(b) shows results of numerical calculations of the effective diameter as a function of the charge parameter $q$. For $q \gtrsim 1$, there is a smooth transition to the theoretical needle limit of Ref. [31], where $\kappa L \gg 1$. Conversely, this is not the case for $q \lesssim 1$, due to the fact that the approximations leading to Eq. (15) do not give the correct effective excluded volume for small values of $q$ and (nearly) parallel rods. More investigations need to be made into this regime.

In conclusion, we have numerically studied the second virial coefficient of short charged rods dispersed in an electrolyte, presuming pairwise screened-Coulomb interactions between the line-charge segments of the rods. The control parameters of interest are the hard-core length $L$ and diameter $D$, the Debye screening length of the medium $\kappa^{-1}$, and the charge parameter $q$. The main resulting quantities are the effective diameter $D_{\text {eff }}$ and length $L_{\text {eff }}$ of the rods. By a mapping onto an effective hard-core system-for which the sequence of phases between the dilute isotropic phase and the dense crystalline phase is known for all aspect ratios-we predict the relations between control parameters and the expected phase sequence explicitly. We have also constructed a simplified model, based on the diameter $\bar{D}(\gamma)$ of Eq. (19), which reproduces the numerical results accurately at the expense of much less computational effort. This model is particularly successful in the regime of large effective aspect ratios $\left(L_{\text {eff }} / D_{\text {eff }}>1\right)$ and small ratios of the rod length to the screening length $(\kappa L<1)$.

An important result of this work is that highly charged short rods at low salt concentrations (i.e., at strong Coulomb couplings) have a strong tendency to form plastic crystals upon compression. The plasticity stems from the large effective diameter, which make the rods behave essentially as inflated repulsive spheres with only small nonspherical interactions that are too weak to cause orientational ordering in the crystalline phase. This finding could be important in the study of silica or gold nanorods, that have reasonably large hard-core aspect ratio (such as $L / D \simeq 5$ ). Here, liquid crystalline phases could be expected, but only if the charge on the rods is small enough.

\section{ACKNOWLEDGMENTS}

It is a pleasure to thank Ahmet Demirörs for explaining his preliminary experimental results on charged dumbbells.

\section{APPENDIX A: THE PAIR INTERACTION OF TWO CHARGED RODS}

The pair interaction of two charged rods is given by Eq. (2), where we assume that the electrostatic interaction is determined by integrating over pairs of effective line-charge elements interacting with the screened Coulomb potential. The distance between these pairs is given by a superposition 
of the relative position of the rods and the combination of the position of the line elements along both rods. Since the integral in Eq. (2) cannot be calculated analytically, we try to simplify the calculation. By expanding the integrand in spherical harmonics, we obtain terms that factorize into two functions of the respective positions (using a special case of the expression in Gradshteyn and Ryzhik [43] for imaginary arguments, or the expression in Abramowitz and Stegun [44])

$$
\begin{aligned}
\frac{\exp [-|\mathbf{r}-\mathbf{s}|]}{|\mathbf{r}-\mathbf{s}|} & =\sum_{l=0}^{\infty}(2 l+1) k_{l}(r) P_{l}(\hat{\mathbf{r}} \cdot \hat{\mathbf{s}}) i_{l}(s) \quad \text { for } r>s, \\
& =4 \pi \sum_{l=0}^{\infty} \sum_{m=-l}^{+l} k_{l}(r) Y_{l, m}(\hat{\mathbf{r}}) i_{l}(s) Y_{l, m}^{*}(\hat{\mathbf{s}}),
\end{aligned}
$$

where $i_{l}$ and $k_{l}$ are the modified spherical Bessel functions of the first and second kind, respectively. These functions are given by

$$
\begin{aligned}
i_{l}(x) & =\sqrt{\frac{\pi}{2 x}} I_{l+1 / 2}(x), \\
k_{l}(x) & =\sqrt{\frac{2}{\pi x}} K_{l+1 / 2}(x),
\end{aligned}
$$

where $I_{\nu}$ and $K_{\nu}$ are the modified (cylindrical) Bessel functions of the first and second kind, respectively. The Legendre polynomials $P_{l}$ are expanded into spherical harmonics $Y_{l, m}$ using the famous addition theorem. We use the notation where $r=|\mathbf{r}|$, and $\hat{\mathbf{r}}=\mathbf{r} / r$. Finally, the asterisk “*” denotes complex conjugation. The unit vector as given in the arguments of each of the spherical harmonic functions should be interpreted as the two angles in spherical coordinates with respect to an arbitrarily chosen reference frame. Since the Legendre polynomials of the dot product of the two orientations are independent of this choice, so is the sum over $m$ of the product of the two spherical harmonics. The expansion can be understood by the fact that the left-hand side is a Green's function in the case of linear screening and a vanishing potential at infinity. This Green's function can be expanded in Legendre polynomials such that it is the solution of an infinite set of ordinary differential equations instead of one partial differential equation. These equations exactly yield the modified spherical Bessel functions.

We note that one could consider rewriting the expression of the pair potential in rotational invariants (as used in Ref. [40]). These are functions of three orientations, including a sum over $m$ of a product of three spherical harmonic functions multiplied by Clebsch-Gordon coefficients. They form a complete set of orthogonal functions dependent only on the relative orientations of $\hat{\mathbf{r}}, \hat{\omega}$, and $\hat{\omega}^{\prime}$ with respect to each other. However, it turns out that in our case these are not really helpful. Alternatively, one could consider a resummation of the expansion in spherical harmonics, such that each term has a faster asymptotic decay than the previous term. This is not the case here, since each Bessel function $k_{l}$ has the same asymptotic decay as $k_{0}$ [24].

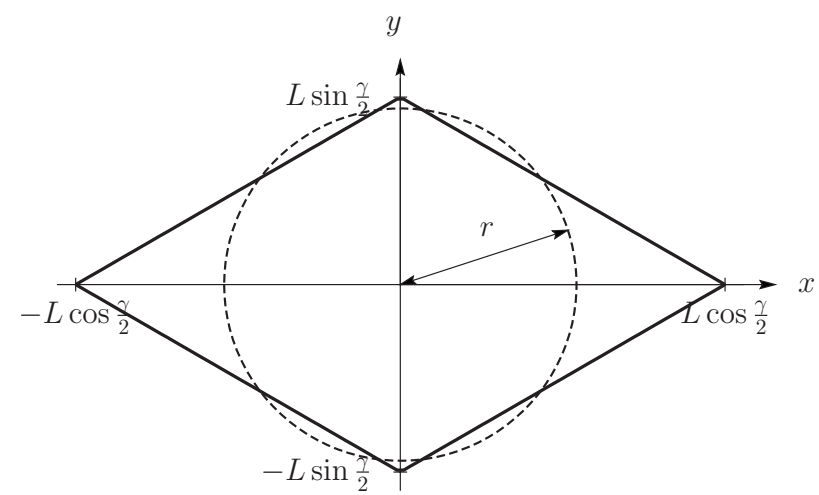

FIG. 8. Illustration of the domain of integration of the superposition of the positions of two line elements. The dashed circle of radius $r$ divides the parallelogram into two domains.

\section{APPENDIX B: DOMAINS OF INTEGRATION}

The integration over line elements of both rods in Eq. (2) is in fact an integration with respect to the vector $l \hat{\omega}-l^{\prime} \hat{\omega}^{\prime}$ over a parallelogram-shaped area in the plane tangent to both rod orientations. This area is illustrated in Fig. 8. There is a straightforward choice for the reference frame and a substitution of variables,

$$
\begin{gathered}
\hat{\omega}=\left(\cos \frac{\gamma}{2}, \sin \frac{\gamma}{2}, 0\right), \\
\hat{\omega}^{\prime}=\left(\cos \frac{\gamma}{2},-\sin \frac{\gamma}{2}, 0\right), \\
l \hat{\omega}-l^{\prime} \hat{\omega}^{\prime}=(\rho \cos \varphi, \rho \sin \varphi, 0),
\end{gathered}
$$

where $\gamma$ is the angle between the two rod orientations. The polar coordinates $\rho$ and $\varphi$ describe the same plane as $l$ and $l^{\prime}$. These coordinates-where $\rho$ is multiplied by the inverse screening length $\kappa$ to make it dimensionless-replace the vector $\mathbf{s}$ in the expansion (A1). Also, the coordinate $r$ is multiplied by $\kappa$. The parallelogram can be cut up into four equivalent pieces, keeping only the terms in the expansion where $l$ and $m$ are both even. The integral boundaries of the first quadrant $(0 \leqslant \varphi \leqslant \pi / 2)$ satisfy

$$
0 \leqslant \rho \leqslant \rho_{\max }(\varphi) \equiv \frac{L \sin \gamma}{2 \sin (\varphi+\gamma / 2)} .
$$

It is important to note that the precise form of the expansion of the integrant of Eq. (2) can vary as a function of $\rho$, because $k_{l}$ and $i_{l}$ switch roles when $\rho>r$. We shall split the result of our expansion into each order in $l$ and $m$, to be examined separately. We write

$$
\begin{aligned}
\beta V_{\mathrm{e}}\left(r, \theta, \phi ; \hat{\omega}, \hat{\omega}^{\prime}\right)= & \kappa l_{\mathrm{B}} \lambda^{2} \underbrace{\sum_{l=0}^{\infty} \sum_{m=-l}^{+l} \frac{(-1)^{(l+m) / 2}(2 l+1)(l-m) !}{2^{l}\left(\frac{l+m}{2}\right) !\left(\frac{l-m}{2}\right) !}}_{l, m \text { even }} \\
& \times \mathcal{A}_{l, m}(r ; \gamma) P_{l, m}(\cos \theta) \cos (m \phi), \quad(\mathrm{B} 5)
\end{aligned}
$$

where $P_{l, m}$ are the associated Legendre functions. We have used that for $l$ and $m$ both even 


$$
\begin{aligned}
& \frac{Y_{l, m}(\theta, \phi)+Y_{l,-m}(\theta, \phi)}{2} \\
& \quad=\sqrt{\frac{2 l+1}{4 \pi} \frac{(l-m) !}{(l+m) !}} P_{l, m}(\cos \theta) \cos (m \phi),
\end{aligned}
$$

and

$$
\begin{aligned}
& \frac{1}{2}\left[Y_{l, m}^{*}\left(\vartheta=\frac{\pi}{2}, \varphi\right)+Y_{l,-m}^{*}\left(\vartheta=\frac{\pi}{2}, \varphi\right)\right] \\
& \quad=(-1)^{(l+m) / 2} \sqrt{\frac{2 l+1}{4 \pi}} \frac{\sqrt{(l+m) !(l-m) !}}{2^{l}\left(\frac{l+m}{2}\right) !\left(\frac{l-m}{2}\right) !} \cos (m \varphi) .
\end{aligned}
$$

The expression for $\mathcal{A}_{l, m}(r ; \gamma)$ in Eq. (B5) is an integral over the four equivalent quadrants of the product of two modified spherical Bessel functions, together with $\cos (m \varphi)$. It is given by

$$
\mathcal{A}_{l, m}(r ; \gamma)=\frac{4}{\sin \gamma} \int_{0}^{\pi / 2} d \varphi \cos (m \varphi) \mathcal{B}_{l}(r ; \varphi, \gamma)
$$

where

$$
\mathcal{B}_{l}(r ; \varphi, \gamma)=k_{l}(\kappa r) \int_{0}^{\rho_{\max }(\varphi)} d \rho \rho i_{l}(\kappa \rho)
$$

for $r>\rho_{\max }(\varphi)$, and

$$
\mathcal{B}_{l}(r ; \varphi, \gamma)=k_{l}(\kappa r) \int_{0}^{r} d \rho \rho i_{l}(\kappa \rho)+i_{l}(\kappa r) \int_{r}^{\rho_{\max }(\varphi)} d \rho \rho k_{l}(\kappa \rho)
$$

for $r<\rho_{\max }(\varphi)$.

Let us have another look at Fig. 8. The dashed circle indicates the value for which the variables $r$ and $s$ in Eq. (A1) switch (in this case $r$ and $s$ are replaced by $\kappa r$ and $\kappa \rho$ ). Consider the first quadrant (i.e., the upper right-hand corner). Let us also assume $\gamma<\pi / 2$, such that $\cos (\gamma / 2)>\sin (\gamma / 2)$. In the end, we will calculate the effective excluded volume for $0<\gamma<\pi$, but this expression will turn out to be symmetric in $\gamma \leftrightarrow \pi-\gamma$ (due to up-down symmetry) so we need only to consider the first half of this interval. The integral boundary for $\rho$ is given as a function of $\varphi$ by the value of $\rho$ on the boundary of the parallelogram. However, the form of $\mathcal{B}_{l}$ changes when the boundary of the parallelogram intersects with the circle of radius $r$. Therefore-depending on the value of $r$-we have one to three domains for $\mathcal{B}_{l}$ as a function of $\varphi$

$$
\begin{gathered}
\varphi \in\left[0, \frac{\pi}{2}\right], \quad \text { for } r<\frac{L \sin \gamma}{2}, \\
\varphi \in[0, \alpha(r)],[\alpha(r), \beta(r)],\left[\beta(r), \frac{\pi}{2}\right], \\
\text { for } \frac{L \sin \gamma}{2}<r<L \sin \frac{\gamma}{2},
\end{gathered}
$$

$$
\begin{gathered}
\varphi \in[0, \alpha(r)],\left[\alpha(r), \frac{\pi}{2}\right], \quad \text { for } L \sin \frac{\gamma}{2}<r<L \cos \frac{\gamma}{2}, \\
\varphi \in\left[0, \frac{\pi}{2}\right], \quad \text { for } r>L \cos \frac{\gamma}{2},
\end{gathered}
$$

where

$$
\begin{gathered}
\alpha(r)=\arcsin \left(\frac{L \sin \gamma}{2 r}\right)-\frac{\gamma}{2}, \\
\beta(r)=\pi-\arcsin \left(\frac{L \sin \gamma}{2 r}\right)-\frac{\gamma}{2}
\end{gathered}
$$

are the angles for which the circle intersects the boundary of the parallelogram. For each of the domains (i.e., subsets of $[0, \pi / 2])$, there is a corresponding circle segment and a corresponding expression for $\mathcal{B}_{l}$. If the circle segment lies in the interior of the parallelogram, we must distinguish between the interval where $\rho$ is smaller than $r$ and vice versa, and therefore we use Eq. (B10). When the circle segment of this domain lies outside of the parallelogram, we use Eq. (B9). Since $\mathcal{A}_{l, m}$ contains an integral of $\varphi$ over the interval $[0, \pi / 2]$, we split it into as many pieces as there are domains, using the corresponding expression for the integrant $\mathcal{B}_{l}$ in each domain.

\section{APPENDIX C: THE LIMIT FOR PARALLEL RODS}

In principle, calculations of the effective excluded volume for parallel rods involves the limit $\gamma \rightarrow 0$ of Eqs. (B8)-(B12). To obtain the correct result, one has to take care to perform the limit correctly in each expression, which is not straightforward. It is much easier to re-evaluate the expressions in this limit analytically, starting with Eqs. (B1)-(B3). We use the same reference frame, but a different substitution of variables

$$
\begin{gathered}
\hat{\omega}=(1,0,0), \\
\hat{\omega}^{\prime}=(1,0,0), \\
l \hat{\omega}-l^{\prime} \hat{\omega}^{\prime}=( \pm x, 0,0),
\end{gathered}
$$

where $x=\left|l-l^{\prime}\right|$. Now the integration is performed over relative positions of two points on a single line. Half of the combinations is positive $\left(l>l^{\prime}\right)$, the other half is negative $\left(l<l^{\prime}\right)$. The integration boundaries of either set is given by

$$
0 \leqslant x \leqslant L .
$$

The length over which each combination $l, l^{\prime}$ is realized, for a certain value of $x$, is given by $L-x$. In accordance with the previous expressions, we define the integral $\mathcal{A}_{l, m}$ for parallel rods as

$$
\mathcal{A}_{l, m}(r ; \gamma=0)=2 k_{l}(\kappa r) \int_{0}^{L} d x(L-x) i_{l}(\kappa x)
$$

for $r>L$, and 


$$
\begin{aligned}
\mathcal{A}_{l, m}(r ; \gamma=0)= & 2 k_{l}(\kappa r) \int_{0}^{r} d x(L-x) i_{l}(\kappa x) \\
& +2 i_{l}(\kappa r) \int_{r}^{L} d x(L-x) k_{l}(\kappa x)
\end{aligned}
$$

for $r<L$. Note that the expressions are independent of $m$.

\section{APPENDIX D: NOTATIONS, INTEGRALS, AND TAYLOR SERIES EXPANSIONS}

In order to calculate the integral $\mathcal{A}_{l, m}$, we first need to calculate the integral $\mathcal{B}_{l}$ by performing the integration-with respect to the radial coordinate $\rho$-in Eqs. (B9) and (B10). Because the calculations are rather intricate, we develop here a short-hand notation to allow for relatively compact expressions. Introducing the notation

$$
\begin{aligned}
& \mathcal{I}_{l}(z)=\int_{0}^{z} d x x i_{l}(x), \\
& \mathcal{K}_{l}(z)=\int_{z}^{\infty} d x x k_{l}(x),
\end{aligned}
$$

we can rewrite $\mathcal{B}_{l}$ as

$$
\kappa^{2} \mathcal{B}_{l}(r ; \varphi, \gamma)= \begin{cases}k_{l}(\kappa r) \mathcal{I}_{l}\left[\kappa \rho_{\max }(\varphi)\right] & \text { for } r>\rho_{\max }(\varphi), \\ k_{l}(\kappa r) \mathcal{I}_{l}(\kappa r)+i_{l}(\kappa r) \mathcal{K}_{l}(\kappa r)-i_{l}(\kappa r) \mathcal{K}_{l}\left[\kappa \rho_{\max }(\varphi)\right] & \text { for } r<\rho_{\max }(\varphi)\end{cases}
$$

Unfortunately, there is no (easy) way to write down the expressions in Eqs. (D1) and (D2) explicitly for arbitrary $l$. However, one can give explicit expressions (necessary for our calculations) for $l=0,2,4$. First, the modified spherical Bessel functions

$$
\begin{gathered}
i_{0}(z)=\frac{\sinh (z)}{z}, \\
i_{2}(z)=\frac{\left(z^{2}+3\right) \sinh (z)-3 z \cosh (z)}{z^{3}}, \\
i_{4}(z)=\frac{\left(z^{4}+45 z^{2}+105\right) \sinh (z)-\left(10 z^{3}+105 z\right) \cosh (z)}{z^{5}}, \\
k_{0}(z)=\frac{\exp (-z)}{z}, \\
k_{4}(z)=\frac{\left(z^{4}+10 z^{3}+45 z^{2}+105 z+105\right) \exp (-z)}{z^{5}}
\end{gathered}
$$

Next, their integrals

$$
\begin{gathered}
\mathcal{I}_{0}(z)=\cosh (z)-1, \\
\mathcal{I}_{2}(z)=\frac{z \cosh (z)-3 \sinh (z)}{z}+2,
\end{gathered}
$$

$$
\begin{gathered}
\mathcal{I}_{4}(z)=\frac{\left(z^{3}+35 z\right) \cosh (z)-\left(10 z^{2}+35\right) \sinh (z)}{z^{3}}-\frac{8}{3}, \\
\mathcal{K}_{0}(z)=\exp (-z) \\
\mathcal{K}_{2}(z)=\frac{(z+3) \exp (-z)}{z} \\
\mathcal{K}_{4}(z)=\frac{\left(z^{3}+10 z^{2}+35 z+35\right) \exp (-z)}{z^{3}}
\end{gathered}
$$

Unfortunately, we cannot perform the subsequent integration-with respect to the angular coordinate $\varphi$-in Eq. (B8) analytically, when we try to calculate $\mathcal{A}_{l, m}$. Therefore, we use the series expansions (for even $l$ )

$$
\begin{aligned}
\mathcal{I}_{2 n}(z) & =2^{2 n} \sum_{k=0}^{\infty} \frac{(2 n+k) ! z^{2 n+2 k+2}}{(2 n+2 k+2)(4 n+2 k+1) ! k !}, \quad \text { (D16) } \\
\mathcal{K}_{2 n}(z)= & (-1)^{n} \frac{\left(2^{n} n !\right)^{2}}{(2 n) !}-\frac{1}{2^{2 n}} \sum_{k=1}^{2 n} \frac{(-1)^{k}(2 k) ! z^{2 n-2 k+1}}{(2 n-2 k+1)(2 n-k) ! k !} \\
& -\frac{1}{2^{2 n}} \sum_{k=0}^{\infty} \frac{k ! z^{2 n+2 k+1}}{(2 n+2 k+1)(2 n+k) !(2 k) !} \\
& +2^{2 n} \sum_{k=0}^{\infty} \frac{(2 n+k) ! z^{2 n+2 k+2}}{(2 n+2 k+2)(4 n+2 k+1) ! k !} .
\end{aligned}
$$

Finally, we define the specific combination

$$
\mathcal{C}_{l}(\kappa r)=k_{l}(\kappa r) \mathcal{I}_{l}(\kappa r)+i_{l}(\kappa r) \mathcal{K}_{l}(\kappa r),
$$

which turns out to be given by a relatively simple expression (for even $l$ ), 


$$
\begin{aligned}
\mathcal{C}_{2 n}(\kappa r)= & \frac{(n !)^{2}}{(2 n) !} \sum_{k=0}^{n} \frac{(-1)^{k}(2 n+2 k) !}{(n+k) !(n-k) !(\kappa r)^{2 k+1}} \\
& -(-1)^{n} \frac{\left(2^{n} n !\right)^{2}}{(2 n) !} k_{2 n}(\kappa r),
\end{aligned}
$$

such that

$$
\begin{gathered}
\mathcal{C}_{0}(z)=\frac{1}{z}-\frac{\exp (-z)}{z}, \\
\mathcal{C}_{2}(z)=\frac{z^{2}-6}{z^{3}}+2 \frac{\left(z^{2}+3 z+3\right) \exp (-z)}{z^{3}}, \\
\mathcal{C}_{4}(z)=\frac{z^{4}-20 z^{2}+280}{z^{5}} \\
-\frac{8}{3} \frac{\left(z^{4}+10 z^{3}+45 z^{2}+105 z+105\right) \exp (-z)}{z^{5}} .
\end{gathered}
$$

(D22)

Note that in each expression the first term cancels the divergence of the second term in the limit where $z \rightarrow 0$. Hence, this limit is given by

$$
\mathcal{C}_{2 n}(0)=\delta_{n, 0} .
$$

This property is also reflected in the series expansionuseful for calculations for small $\kappa r$-given by

$$
\begin{aligned}
\mathcal{C}_{2 n}(\kappa r)= & (-1)^{n} \frac{\left(2^{n} n !\right)^{2}}{(2 n) !} \\
& \times \frac{\sqrt{\pi}}{2} \sum_{k=0}^{\infty} \frac{1}{\Gamma\left(\frac{k+2 n+3}{2}\right) \Gamma\left(\frac{k-2 n+2}{2}\right)}\left(\frac{-\kappa r}{2}\right)^{k} .
\end{aligned}
$$

Note that the terms for even $k<2 n$ have vanishing coefficients.

The limit of parallel rods has a different set of expressions. Therefore, we define an additional notation

$$
\begin{gathered}
\mathcal{J}_{l}(z)=\int_{0}^{z} d x \frac{z-x}{z} i_{l}(x), \\
\mathcal{L}_{l}(z)=\int_{z}^{\infty} d x \frac{z-x}{z} k_{l}(x) .
\end{gathered}
$$

In this way, we split each integral in Eq. (C6) in two parts

$$
\kappa^{2} \mathcal{A}_{l, m}(r ; \gamma=0)= \begin{cases}2 \kappa L k_{l}(\kappa r) \mathcal{J}_{l}(\kappa L) & \text { for } r>L, \\ 2 \frac{L-r}{r} \mathcal{C}_{l}(\kappa r)+2 \kappa L k_{l}(\kappa r) \mathcal{J}_{l}(\kappa r)+2 \kappa L i_{l}(\kappa r)\left[\mathcal{L}_{l}(\kappa r)-\mathcal{L}_{l}(\kappa L)\right] & \text { for } r<L .\end{cases}
$$

Evaluation of these integrals result in slightly more complicated expressions, when compared to the expressions for $\mathcal{I}$ and $\mathcal{K}$ in Eqs. (D10)-(D15),

$$
\begin{gathered}
\mathcal{J}_{0}(z)=\operatorname{shi}(z)+\frac{1}{z}-\frac{\cosh (z)}{z}, \\
\mathcal{J}_{2}(z)=-\frac{1}{2} \operatorname{shi}(z)-\frac{2}{z}+\frac{z \cosh (z)+3 \sinh (z)}{2 z^{2}}, \\
\mathcal{J}_{4}(z)=\frac{3}{8} \operatorname{shi}(z)+\frac{8}{3 z} \\
-\frac{\left(3 z^{3}+70 y\right) \cosh (z)-\left(5 z^{2}+70\right) \sinh (z)}{8 z^{4}},
\end{gathered}
$$

where

$$
\operatorname{shi}(z)=\int_{0}^{z} d x \frac{\sinh (x)}{x},
$$

is the hyperbolic sine integral.

$$
\begin{gathered}
\mathcal{L}_{0}(z)=\Gamma(0, z)-\frac{\exp (-z)}{z}, \\
\mathcal{L}_{2}(z)=-\frac{1}{2} \Gamma(0, z)+\frac{(z-3) \exp (-z)}{2 z^{2}}, \\
\mathcal{L}_{4}(z)=\frac{3}{8} \Gamma(0, z)-\frac{\left(3 z^{3}+5 z^{2}+70 y+70\right) \exp (-z)}{8 z^{4}} .
\end{gathered}
$$

One now has the exact solutions for $\mathcal{A}_{l, m}$-in the case of parallel rods-up to $l=4$. However, we need the expressions in Eqs. (D28)-(D34) to provide a well-defined limit for $\gamma$ $\rightarrow 0$, to use in combination with the expressions for arbitrary orientations [i.e., the series expansions in Eqs. (D16), (D17), and (D19)]. Therefore, it will be convenient to also have these expressions in the form of a series expansion,

$$
\mathcal{J}_{2 n}(z)=2^{2 n} \sum_{k=0}^{\infty} \frac{(2 n+k) ! z^{2 n+2 k+1}}{(2 n+2 k+1)(2 n+2 k+2)(4 n+2 k+1) ! k !},
$$




$$
\begin{aligned}
\mathcal{L}_{2 n}(z)= & (-1)^{n} \frac{(2 n) !}{\left(2^{n} n !\right)^{2}}\left(1+\sum_{k=1}^{2 n} \frac{1}{k}-\gamma_{\mathrm{E}}-\ln (z)\right)-(-1)^{n} \frac{\left(2^{n} n !\right)^{2}}{(2 n) !} \frac{1}{z}-\frac{1}{2^{2 n}} \sum_{k=0, k \neq n}^{2 n} \frac{(-1)^{k}(2 k) ! z^{2 n-2 k}}{(2 n-2 k)(2 n-2 k+1)(2 n-k) ! k !} \\
& -\frac{1}{2^{2 n}} \sum_{k=1}^{\infty} \frac{k ! z^{2 n+2 k}}{(2 n+2 k)(2 n+2 k+1)(2 n+k) !(2 k) !}+2^{2 n} \sum_{k=0}^{\infty} \frac{(2 n+k) ! z^{2 n+2 k+1}}{(2 n+2 k+1)(2 n+2 k+2)(4 n+2 k+1) ! k !} .
\end{aligned}
$$

\section{APPENDIX E: TRUNCATION AND SOME EXAMPLES OF EXPRESSIONS}

In principle, the calculation of each of the terms in Eq. (B5) (i.e., each order of $l$ and $m$ ) involves an infinite series expansion in $\kappa L$. We will restrict our calculations to $l=0,2$, and 4 , and truncate each series expansion. Since the shape of the integration domain of $\mathcal{A}_{l, m}$ is a parallelogram with sides of length $L$, we divide out a factor $L^{2}$ to make both $\mathcal{A}_{l, m}$ and $\mathcal{B}_{l}$ dimensionless [i.e., we calculate $\kappa^{2} \mathcal{A}_{l, m} /(\kappa L)^{2}$ and $\kappa^{2} \mathcal{B}_{l} /(\kappa L)^{2}$ ]. This factor $L^{2}$ is combined with the prefactor $\kappa l_{\mathrm{B}} \lambda^{2}$ in Eq. (B5). From the definition of the charge parameter $q$, we can write the result as an overall prefactor $q \kappa^{2} L^{2}$. The truncated expansion is defined as the expansion up to fourth order in $\kappa L$ of the expression where this prefactor is taken out. This means that we determine the series expansions of the expressions in Eqs. (D3) and (D27), after we divide by a factor $(\kappa L)^{2}$. We give some examples of the calculated expressions for $l=0$ and $m=0$, where we explicitly make the distinction between four domains in $r$. For $r<\frac{L \sin \gamma}{2}$,

$$
\begin{aligned}
\kappa^{2} \mathcal{A}_{0,0}(r ; \gamma)= & \frac{4}{\sin \gamma} \int_{0}^{\pi / 2} d \varphi\left\{\mathcal{C}_{0}(\kappa r)-i_{0}(\kappa r) \mathcal{K}_{0}\left[\kappa \rho_{\max }(\varphi)\right]\right\} \\
\simeq & \frac{2 \pi}{\sin \gamma}\left(\frac{1}{\kappa r}-\frac{\exp (-\kappa r)}{\kappa r}-\frac{\sinh (\kappa r)}{\kappa r}\right)+\frac{\sinh (\kappa r)}{\kappa r} \kappa^{2} L^{2}\left[-\ln \left(\tan \frac{\gamma}{4} \tan \frac{\pi-\gamma}{4}\right)\left(\frac{2}{\kappa L}+\frac{\kappa L \sin ^{2} \gamma}{24}+\frac{\kappa^{3} L^{3} \sin ^{4} \gamma}{2560}\right)\right. \\
& +\sqrt{1+\sin \gamma}(2-\sin \gamma) \frac{\kappa L}{12}+\sqrt{1+\sin \gamma}\left(16-8 \sin \gamma+2 \sin ^{2} \gamma-3 \sin ^{3} \gamma\right) \frac{\kappa^{3} L^{3}}{3840}-1-\frac{\kappa^{2} L^{2}}{36} \\
& \left.-\left(7+5 \cos ^{2} \gamma\right) \frac{\kappa^{4} L^{4}}{21600}\right] .
\end{aligned}
$$

The next domain is $\frac{L \sin \gamma}{2}<r<L \sin \frac{\gamma}{2}$, where the expression is a lot more involved, because the integration interval $[0, \pi / 2]$ is split into three pieces,

$$
\begin{aligned}
\kappa^{2} \mathcal{A}_{0,0}(r ; \gamma)= & \frac{4}{\sin \gamma}\left\{\int_{0}^{\alpha(\kappa r)} d \varphi\left\{\mathcal{C}_{0}(\kappa r)-i_{0}(\kappa r) \mathcal{K}_{0}\left[\kappa \rho_{\max }(\varphi)\right]\right\}+\int_{\alpha(\kappa r)}^{\beta(\kappa r)} d \varphi k_{0}(\kappa r) \mathcal{I}_{0}\left[\kappa \rho_{\max }(\varphi)\right]\right. \\
& \left.+\int_{\beta(\kappa r)}^{\pi / 2} d \varphi\left\{\mathcal{C}_{0}(\kappa r)-i_{0}(\kappa r) \mathcal{K}_{0}\left[\kappa \rho_{\max }(\varphi)\right]\right\}\right\} \\
\simeq & \frac{4}{\sin \gamma}\left(2 \arcsin (\xi)-\frac{\pi}{2}\right)\left(\frac{1}{\kappa r}-\frac{\exp (-\kappa r)}{\kappa r}-\frac{\sinh (\kappa r)}{\kappa r}\right) \\
& +\frac{\sinh (\kappa r)}{\kappa r} \kappa^{2} L^{2}\left[-\ln \left(\tan \frac{\gamma}{4} \tan \frac{\pi-\gamma}{4}\right)\left(\frac{2}{\kappa L}+\frac{\kappa L \sin ^{2} \gamma}{24}+\frac{\kappa^{3} L^{3} \sin ^{4} \gamma}{2560}\right)+\sqrt{1+\sin \gamma(2-\sin \gamma) \frac{\kappa L}{12}}\right. \\
& +\sqrt{1+\sin \gamma}\left(16-8 \sin \gamma+2 \sin ^{2} \gamma-3 \sin ^{3} \gamma\right) \frac{\kappa^{3} L^{3}}{3840}-1-\frac{\kappa^{2} L^{2}}{36}-\left(7+5 \cos ^{2} \gamma\right) \frac{\kappa^{4} L^{4}}{21600}-\frac{2 r}{L} \operatorname{arctanh}\left(\sqrt{1-\xi^{2}}\right)\left(\frac{2}{\kappa r}\right. \\
& \left.\left.+\xi^{2} \frac{\kappa r}{6}+\xi^{4} \frac{\kappa^{3} r^{3}}{160}\right)-\frac{2 r}{L} \sqrt{1-\xi^{2}}\left(\frac{\kappa r}{6}+\left(3 \xi^{2}+2\right) \frac{\kappa^{3} r^{3}}{480}-1-\left(2 \xi^{2}+1\right) \frac{\kappa^{2} r^{2}}{36}-\left(8 \xi^{4}+4 \xi^{2}+3\right) \frac{\kappa^{4} r^{4}}{5400}\right)\right] \\
& +\frac{\exp (-\kappa r)}{\kappa r} \kappa^{2} L^{2}\left[\frac{2 r}{L} \sqrt{1-\xi^{2}}\left(1+\left(2 \xi^{2}+1\right) \frac{\kappa^{2} r^{2}}{36}+\left(8 \xi^{4}+4 \xi^{2}+3\right) \frac{\kappa^{4} r^{4}}{5400}\right)\right] .
\end{aligned}
$$


We have abbreviated

$$
\xi=\frac{L \sin \gamma}{2 r}
$$

This domain corresponds to the case where the circle of radius $r$ intersects the edge of the parallelogram twice at each quadrant. The following domain corresponds to the case where there is just one intersection per quadrant. Recall that we assume $0<\gamma<\pi / 2$, such that this domain is given by $L \sin \frac{\gamma}{2}<r<L \cos \frac{\gamma}{2}$,

$$
\begin{aligned}
\kappa^{2} \mathcal{A}_{0,0}(r ; \gamma)= & \frac{4}{\sin \gamma}\left\{\int_{0}^{\alpha(\kappa r)} d \varphi\left\{\mathcal{C}_{0}(\kappa r)-i_{0}(\kappa r) \mathcal{K}_{0}\left[\kappa \rho_{\max }(\varphi)\right]\right\}+\int_{\alpha(\kappa r)}^{\pi / 2} d \varphi k_{0}(\kappa r) \mathcal{I}_{0}\left[\kappa \rho_{\max }(\varphi)\right]\right\} \\
\simeq & \frac{4}{\sin \gamma}\left(\arcsin (\xi)-\frac{\gamma}{2}\right)\left(\frac{1}{\kappa r}-\frac{\exp (-\kappa r)}{\kappa r}-\frac{\sinh (\kappa r)}{\kappa r}\right)+\frac{\sinh (\kappa r)}{\kappa r} \kappa^{2} L^{2}\left[-\ln \left(\tan \frac{\gamma}{4}\right)\left(\frac{2}{\kappa L}+\frac{\kappa L \sin { }^{2} \gamma}{24}\right.\right. \\
& \left.+\frac{\kappa^{3} L^{3} \sin ^{4} \gamma}{2560}\right)+\left(\frac{1+\cos \gamma}{2}\right)^{3 / 2} \frac{\kappa L}{6}+\left(\frac{1+\cos \gamma}{2}\right)^{5 / 2}(7-3 \cos \gamma) \frac{\kappa^{3} L^{3}}{960}-\frac{1+\cos \gamma}{2}-\left(\frac{1+\cos \gamma}{2}\right)^{2} \\
& \times(2-\cos \gamma) \frac{\kappa^{2} L^{2}}{36}-\left(\frac{1+\cos \gamma}{2}\right)^{3}\left(7-6 \cos \gamma+2 \cos ^{2} \gamma\right) \frac{\kappa^{4} L^{4}}{5400}-\frac{r}{L} \operatorname{arctanh}\left(\sqrt{1-\xi^{2}}\right)\left(\frac{2}{\kappa r}+\xi^{2} \frac{\kappa r}{6}+\xi^{4} \frac{\kappa^{3} r^{3}}{160}\right) \\
& \left.-\frac{r}{L} \sqrt{1-\xi^{2}}\left(\frac{\kappa r}{6}+\left(3 \xi^{2}+2\right) \frac{\kappa^{3} r^{3}}{480}-1-\left(2 \xi^{2}+1\right) \frac{\kappa^{2} r^{2}}{36}-\left(8 \xi^{4}+4 \xi^{2}+3\right) \frac{\kappa^{4} r^{4}}{5400}\right)\right]+\frac{\exp (-\kappa r)}{\kappa r} \kappa^{2} L^{2} \\
& \times\left[\frac{1-\cos \gamma}{2}+\left(\frac{1-\cos \gamma}{2}\right)^{2}(2+\cos \gamma) \frac{\kappa^{2} L^{2}}{36}+\left(\frac{1-\cos \gamma}{2}\right)^{3}\left(7+6 \cos \gamma+2 \cos ^{2} \gamma\right) \frac{\kappa^{4} L^{4}}{5400}\right. \\
& \left.+\frac{r}{L} \sqrt{1-\xi^{2}}\left(1+\left(2 \xi^{2}+1\right) \frac{\kappa^{2} r^{2}}{36}+\left(8 \xi^{4}+4 \xi^{2}+3\right) \frac{\kappa^{4} r^{4}}{5400}\right)\right] \cdot
\end{aligned}
$$

Finally, the domain where $r>L \cos \frac{\gamma}{2}$ yields a more friendly expression,

$$
\kappa^{2} \mathcal{A}_{0,0}(r ; \gamma)=\frac{4}{\sin \gamma} \int_{0}^{\pi / 2} d \varphi k_{0}(\kappa r) \mathcal{I}_{0}\left[\kappa \rho_{\max }(\varphi)\right] \simeq \frac{\exp (-\kappa r)}{\kappa r} \kappa^{2} L^{2}\left[1+\frac{\kappa^{2} L^{2}}{36}+\left(7+5 \cos ^{2} \gamma\right) \frac{\kappa^{4} L^{4}}{21600}\right]
$$

In the case of parallel rods, we can apply the alternative series expansions, or apply the limit $\gamma \rightarrow 0$ on the last two expressions above. Both yield the following approximations, where for $r<L$

$$
\begin{aligned}
\kappa^{2} \mathcal{A}_{0,0}(r ; \gamma=0)= & 2 \frac{L-r}{r}\left(\frac{1}{\kappa r}-\frac{\exp (-\kappa r)}{\kappa r}\right)+2 \kappa L \frac{\exp (-\kappa r)}{\kappa r}\left(\operatorname{shi}(\kappa r)+\frac{1}{\kappa r}-\frac{\cosh (\kappa r)}{\kappa r}\right)+2 \kappa L \frac{\sinh (\kappa r)}{\kappa r}\left(\Gamma(0, \kappa r)-\frac{\exp (-\kappa r)}{\kappa r}\right. \\
& \left.-\Gamma(0, \kappa L)+\frac{\exp (-\kappa L)}{\kappa L}\right) \simeq 2 \frac{L-r}{r}\left(\frac{1}{\kappa r}-\frac{\exp (-\kappa r)}{\kappa r}\right)+2 \kappa L \frac{\exp (-\kappa r)}{\kappa r} \frac{\kappa r}{2}\left(1+\frac{\kappa^{2} r^{2}}{36}+\frac{\kappa^{4} r^{4}}{1800}\right) \\
& +2 \kappa L \frac{\sinh (\kappa r)}{\kappa r}\left[\ln \left(\frac{L}{r}\right)-\frac{1}{\kappa L} \frac{L-r}{r}+\frac{\kappa r}{2}\left(1-\frac{\kappa r}{6}+\frac{\kappa^{2} r^{2}}{36}-\frac{\kappa^{3} r^{3}}{240}+\frac{\kappa^{4} r^{4}}{1800}\right)-\frac{\kappa L}{2}\left(1-\frac{\kappa L}{6}+\frac{\kappa^{2} L^{2}}{36}-\frac{\kappa^{3} L^{3}}{240}\right.\right. \\
& \left.\left.+\frac{\kappa^{4} L^{4}}{1800}\right)\right]
\end{aligned}
$$

For $r>L$ we obtain

$$
\begin{aligned}
\kappa^{2} \mathcal{A}_{0,0}(r ; \gamma=0) & =2 \kappa L \frac{\exp (-\kappa r)}{\kappa r}\left(\operatorname{shi}(\kappa L)+\frac{1}{\kappa L}-\frac{\cosh (\kappa L)}{\kappa L}\right) \\
& \simeq 2 \kappa L \frac{\exp (-\kappa r)}{\kappa r} \frac{\kappa L}{2}\left(1+\frac{\kappa^{2} L^{2}}{36}+\frac{\kappa^{4} L^{4}}{1800}\right)
\end{aligned}
$$

Likewise, there are expressions for $l=2,4$. These are all used together to create an (approximate) expression for the pair interaction outside of the hard-core exclusion region. We use this pair interaction to numerically calculate the effective excluded volume. This is accomplished by a numerical integration scheme over all different domains of $r$, for given rod orientations. Our approach is fundamentally different from other theoretical work $[24,25]$, in the sense that we apply the interchange of the two positional vectors $\mathbf{r}$ and $l \hat{\omega}-l^{\prime} \hat{\omega}$. We 
have to do this in order to calculate the full integral over $r$, in contrast to the studies in Refs. [24,25], where only a description is given of the pair interaction for rods at large distances.
Conversely, if one considers nonspherical charge distributions on spherical particles, this switch is not needed when introducing rotational invariants.
[1] H. Zocher, Z. Anorg. Allg. Chem. 147, 91 (1925).

[2] F. C. Bawden, N. W. Pirie, J. D. Bernal, and I. Fankuchen, Nature (London) 138, 1051 (1936).

[3] L. Onsager, Ann. N.Y. Acad. Sci. 51, 627 (1949).

[4] S. Fraden, G. Maret, D. L. D. Caspar, and R. B. Meyer, Phys. Rev. Lett. 63, 2068 (1989).

[5] Z. Dogic and S. Fraden, Philos. Trans. R. Soc. London, Ser. A 359, 997 (2001).

[6] M. P. B. van Bruggen, F. M. van der Kooij, and H. N. W. Lekkerkerker, J. Phys.: Condens. Matter 8, 9451 (1996).

[7] E. Snoeks, A. van Blaaderen, T. van Dillen, C. M. van Kats, M. L. Brongersma, and A. Polman, Adv. Mater. (Weinheim, Ger.) 12, 1511 (2000).

[8] P. M. Johnson, C. M. van Kats, and A. van Blaaderen, Langmuir 21, 11510 (2005).

[9] Y. Yin and A. P. Alivisatos, Nature (London) 437, 664 (2005).

[10] H. R. Sheu, M. S. El-Aasser, and J. W. Vanderhoff, J. Polym. Sci. A 28, 629 (1990).

[11] Y.-W. Jun, J.-W. Seo, S. J. Oh, and J. Cheon, Coord. Chem. Rev. 249, 1766 (2005).

[12] C. J. Murphy, T. K. Sau, A. M. Gole, C. J. Orendorff, J. Gao, L. Gou, S. E. Hunyadi, and T. Li, J. Phys. Chem. B 109, 13857 (2005).

[13] C. I. Zoldesi and A. Imhof, Adv. Mater. (Weinheim, Ger.) 17, 924 (2005).

[14] Y.-S. Cho, G.-R. Yi, J.-M. Lim, S.-H. Kim, V. N. Manoharan, D. J. Pine, and S.-M. Yang, J. Am. Chem. Soc. 127, 15968 (2005).

[15] D. J. Kraft, W. S. Vlug, C. M. van Kats, A. van Blaaderen, A. Imhof, and W. K. Kegel, J. Am. Chem. Soc. 131, 1182 (2009).

[16] B. Derjaguin, Trans. Faraday Soc. 35, 203 (1940).

[17] E. J. W. Verwey and J. T. G. Overbeek, Theory of the Stability of Lyophobic Colloids (Elsevier, Amsterdam, 1948).

[18] P. Bolhuis and D. Frenkel, J. Chem. Phys. 106, 666 (1997).

[19] J. A. Cuesta and Y. Martínez-Ratón, J. Chem. Phys. 107, 6379 (1997).

[20] Y. Martínez-Ratón and J. A. Cuesta, J. Chem. Phys. 111, 317 (1999).

[21] R. Eppenga and D. Frenkel, Mol. Phys. 52, 1303 (1984).

[22] A. Esztermann, H. Reich, and M. Schmidt, Phys. Rev. E 73,
011409 (2006).

[23] P. Pfleiderer and T. Schilling, Phys. Rev. E 75, 020402(R) (2007).

[24] R. Ramirez and R. Kjellander, J. Chem. Phys. 125, 144110 (2006).

[25] D. Chapot, L. Bocquet, and E. Trizac, J. Chem. Phys. 120, 3969 (2004).

[26] A. Poniewierski and R. Hołyst, Phys. Rev. A 41, 6871 (1990).

[27] A. M. Somoza and P. Tarazona, Phys. Rev. A 41, 965 (1990).

[28] H. Graf and H. Löwen, J. Phys.: Condens. Matter 11, 1435 (1999).

[29] J. A. C. Veerman and D. Frenkel, Phys. Rev. A 41, 3237 (1990).

[30] G. J. Vroege and H. N. W. Lekkerkerker, Rep. Prog. Phys. 55, 1241 (1992).

[31] A. Stroobants, H. N. W. Lekkerkerker, and T. Odijk, Macromolecules 19, 2232 (1986).

[32] C. Vega and P. A. Monson, J. Chem. Phys. 107, 2696 (1997).

[33] M. Marechal and M. Dijkstra, Phys. Rev. E 77, 061405 (2008).

[34] T. Sato and A. Teramoto, Physica A 176, 72 (1991).

[35] I. A. Nyrkova and A. R. Khokhlov, Biophysics (Engl. Transl.) 31, 839 (1986).

[36] I. A. Nyrkova, N. P. Shusharina, and A. R. Khokhlov, Macromol. Theory Simul. 6, 965 (1997).

[37] S.-B. Chen and D. L. Koch, J. Chem. Phys. 104, 359 (1996).

[38] I. I. Potemkin, R. E. Limberger, A. N. Kudlay, and A. R. Khokhlov, Phys. Rev. E 66, 011802 (2002).

[39] B. Weyerich, B. D’Aguanno, E. Canessa, and R. Klein, Faraday Discuss. Chem. Soc. 90, 245 (1990).

[40] H. Graf and H. Löwen, Phys. Rev. E 59, 1932 (1999).

[41] A. F. Demirors, A. Imhof, and A. van Blaaderen (private communication).

[42] M. P. Allen, G. T. Evans, D. Frenkel, and B. Mulder, Adv. Chem. Phys. 86, 1 (1993).

[43] I. S. Gradshteyn and I. M. Ryzhik, Table of Integrals, Series, and Products (Academic Press, New York, 1980), Eq. 8.5321.

[44] Handbook of Mathematical Functions, edited by M. Abramowitz and I. A. Stegun (Dover Publications, New York, 1965), Eq. 10.2.35. 